\title{
Intravitreal bevacizumab as an adjunctive therapy before diabetic vitrectomy
}

\author{
Ashraf M El-Batarny \\ Assistant Professor of Ophthalmology, \\ Tanta University, Egypt; Chief of \\ Vitreoretinal Service, Magrabi Eye \\ and Ear Hospital, Muscat, Sultanate \\ of Oman
}

Purpose: To evaluate the effect of intravitreal injection of Avastin, bevacizumab (IVA) on diabetic vitrectomy and on the postoperative course.

Methods: Thirty patients undergoing diabetic vitrectomy were distributed to standard vitrectomy (group 1) or vitrectomy with preoperative IVA (group 2). Bevacizumab was injected 5 to 7 days before surgery. Patients of both groups were matched as much as possible according to surgical indication and preoperative visual acuity. Main outcome measures were the feasibility of surgery and the postoperative complications. Feasibility of surgery was evaluated through recording surgical time, intraoperative bleeding, use of endodiathermy, relaxing retinotomies, use of perfluorocarbon liquid (PFCL) and silicone oil tamponade.

Results: Follow up ranged between 7 and 18 months. There was significant reduction of mean surgical time, bleeding frequency, and diathermy use in group 2 compared to group 1 . The rate of PFCL use and relaxing retinotomies were less in group 2 in comparison to group 1. Gas or air was used in $80 \%$ of patients in group 2 while silicone oil represented $60 \%$ of group 1 . Postoperative visual acuity improvement was highly significant in both groups but the difference in both groups was not statistically significant. Vision improved in $87 \%$ in group 2 and $80 \%$ in group 1. Primary anatomical attachment was achieved in $90.3 \%$ in group 2 and $86.6 \%$ in group 1. Rate of subsequent surgeries and persistent cataract were higher in group 1. Postoperative bleeding was reported in $26.6 \%$ of cases in group 1 and none in group 2 . In group 2 , no complications related to avastin injection or progression of traction were reported during the preoperative period.

Conclusion: Preoperative IVA was helpful in achieving the surgical and anatomical goals by reducing the time of surgery, the intraoperative and postoperative bleeding, and the use of silicone oil with subsequent reduction of second surgery.

Keywords: intravitreal bevacizumab, proliferative diabetic retinopathy, diabetic vitrectomy, vascular endothelial growth factor, diabetic vitreous hemorrhage

\section{Introduction}

The first goal of diabetic vitrectomy is to restore vision. Surgical objectives to achieve this goal are thorough removal of vitreous blood, reattachment of the macula, and removal of cataract. The second and equally important goal of surgery is to stabilize the diabetic neovascular process thereby producing long-term anatomic and visual success. The surgical objectives to accomplish this goal are removal of the vitreous scaffold, removal of the surface fibrovascular proliferation, and endophotocoagulation of the ischemic retina. Eyes in which these surgical objectives are achieved and which have a good 6-month outcome tend to remain stable for many years (Blankenship 1981; Blankenship and Machemer 1985; Meredith 1994). Tractional retinal detachment (TRD) and nonresolving vitreous hemorrhage are common indications for diabetic vitrectomy (Meredith 1994). Intraocular hemorrhage represents a serious event during diabetic vitrectomy. Extensive hemorrhage may prevent the successful conclusion of surgery. The removal of clotted blood may not only extend a preexisting retinal
Correspondence:Ashraf M El-Batarny Magrabi Eye and Ear Hospital, PO Box 5I3, P C I I2, Muscat, Sultanate of Oman Email elbatarny@hotmail.com 
break but also may create new retinal breaks (Lee and Abrams 2001).

The most common indication for reoperation after diabetic surgery is recurrent vitreous hemorrhage. Approximately $60 \%$ of eyes develop recurrent vitreous hemorrhage sometime in the postoperative period (Liggett et al 1987; Packer et al 1989; Meredith 1994). Hemorrhage occurs within the first few days after surgery but may occur months or years later. Two thirds of all hemorrhages occur during the first 6 months (Tolentino et al 1989). Various medications and surgical techniques have been utilized to prevent vitreous hemorrhage. Several interventions has been reported to be successful in preventing hemorrhage in the first few days after surgery, but the percentage of cases with significant hemorrhage 2-4 weeks after surgery has not been changed by any of these approaches. Such interventions included intravenous aminocaproic acid (de Bustros et al 1985a), layering of sodium hyaluronate on areas of severed fibrovascular proliferation (Packer et al 1989), intraocular injection of thrombin (de Bustros et al 1985b), and endolaser photocoagulation (Liggett et al 1987). Air-fluid exchange has not been shown to have long term beneficial effect (Liggett et al 1987). Bevacizumab (Avastin, Genentech Inc., San Francisco, CA), a full length humanized monoclonal antibody to vascular endothelial growth factor (VEGF) was approved by the US Food and Drug Administration (FDA) for the treatment for metastatic colorectal cancer (Manzano et al 2006). Recent reports on the intravitreal injection of Avastin (IVA) showed promise for targeting VEGF-implicated intraocular neovascularization seen in age-related macular degeneration (Avery et al 2006) and proliferative diabetic retinopathy (PDR) (Spaide and Fisher 2006). It has been recently shown to enhance the clearance of vitreous hemorrhage and induce involution of retinal neovascularization, (Avery 2006; Spaide and Fisher 2006; Chen and Park 2006) and anterior segment neovascularization (Avery 2006; Davidorf et al 2006; Oshima et al 2006; Grisanti et al 2006) with no reported complications.

This prospective comparative randomized study was constructed to study the effect of preoperative intravitreal injection of bevacizumab on the procedure of diabetic vitrectomy and on its postoperative course.

\section{Methods}

Thirty patients with PDR undergoing pars plana vitrectomy (PPV) were randomized by diagnosis into 2 groups; group 1 undergoing PPV (15 patients) and group 2 undergoing PPV with preoperative IVA (15 patients) (Table 1). Cases of both groups were matched as possible according to surgical indication and preoperative visual acuity. In group 2, bevacizumab was injected 5 to 7 days prior to surgery. The admission criteria were TRD involving or threatening the macula, TRD plus vitreous hemorrhage, nonclearing vitreous hemorrhage of at least one month and massive pre-retinal subhyaloid bleeding covering the posterior pole. Full preoperative and postoperative evaluation included best-corrected visual acuity (VA), fundus biomicroscopy, gonioscopy, and ultrasonography if the fundus could not be seen. All the patients were examined postoperatively at 1, 7 days, 2 weeks, 1 month, and thereafter monthly up to the end of follow-up. The main outcome measures were feasibility of surgery and postoperative complications. Feasibility of surgery was evaluated through recording the time of surgery, frequency of intraoperative bleeding, frequency of use of endodiathermy, relaxing retinotomies, use of perfluorocarbon liquid (PFCL), and use of silicone oil tamponade. The time of surgery was calculated as the time from the entry of the probes in the vitreous cavity till the closure of the vitrectomy ports. This time was calculated by revision of the video recordings of surgeries. Additional procedures as elective phaco-surgery

Table I Age, follow-up, and distribution of cases according to surgical diagnosis

\begin{tabular}{|c|c|c|c|c|}
\hline & $\begin{array}{l}\text { Group I } \\
\text { PPV }\end{array}$ & $\begin{array}{l}\text { Group } 2 \\
\text { Avastin + PPV }\end{array}$ & $\begin{array}{l}\text { P value } \\
(w)\end{array}$ & $\begin{array}{l}\text { P value } \\
\text { (chi) }\end{array}$ \\
\hline Age, $y$, mean (SD)/(range) & $46(12) /(24-65)$ & $44(11) /(23-57)$ & 0.7265 & \\
\hline \multicolumn{5}{|l|}{ Indication of surgery, n (\%) } \\
\hline TRD & $4(26.7)$ & $4(26.7)$ & & I \\
\hline TRD + vitreous Hge. & $6(40)$ & $8(53.3)$ & & 0.7152 \\
\hline Preretinal Hge. & $2(\mid 3.3)$ & I (6.7) & & 1 \\
\hline Dense vitreous Hge. & $3(20)$ & $2(13.3)$ & & I \\
\hline Follow-up, mo., mean (SD)/(range) & II.7 (3.0)/(7-17) & $12.9(3.2) /(7-18)$ & 0.2869 & \\
\hline
\end{tabular}

Abbreviations: SD, standard deviation;TRD, tractional retinal detachment; Hge, hemorrhage; Y, years; mo, months; PPV, pars plana vitrectomy; w, Wilcoxon two-sample test; chi, chi square test. 
as well as periods of time wasted outside the globe such as during exchange of the infusion bottle or getting a new light probe were excluded from the time of surgery and the actual surgical time was calculated by adding the time periods during which the instruments were inside the globe through strict revision of the video tapes. The decision to use gasair mixture or air or silicone oil was taken intraoperatively. The rationale for using silicone oil were; multiple breaks, relaxing retinotomies, and highly active retinal neovascularization with significant intraoperative bleeding predictive of postoperative hemorrhage and in cases requiring re-PPV. Postoperative complications including corneal erosions, recurrent vitreous hemorrhage, reproliferation, cataract, rubeosis, neovascular glaucoma, retinal detachment, were recorded. Complications related to IVA including increase of traction and any systemic side effects were recorded. All cases completed a minimum follow up of 6 months.

\section{Bevacizumab injection}

$1.25 \mathrm{mg}$ in $0.05 \mathrm{~mL}$ bevacizumab (Avastin, Genentech) was injected intravitreally under complete sterile preparation in the operating theater in the usual sterile fashion (Aiello et al 2004) using topical anesthesia. Prophylactic topical antibiotic (moxifloxacine) was given for 3 days postinjection.

\section{PPV}

Standard 3-port PPV utilizing Alcon Accurus surgical system. All surgeries were performed by the same surgeon. Preretinal fibrovascular membranes were removed using different techniques including membrane peeling, segmentation, delamination and en bloc dissection. High speed 23 gauge cutters up to 2500 cuts/minute were used to cut membranes very close to the retina. Bimanual technique was used whenever needed to safely remove membranes using the 27-gauge Eckardt twin light chandelier (D.O.R.C). PFCL was used to stabilize the retina during membrane dissection whenever required. Complete panretinal photocoagulation (PRP) was done at the end of surgery. Single raw of cryotherapy is applied to the anterior retinal and around the sclerotomy sites in an attempt to quell fibrovascular ingrowth and subsequent hemorrhage. No internal limiting membrane peeling was done in any of the surgeries. The internal tamponade used was decided intraoperatively, either air, Sf6/air mixture 20\%, or silicone oil.

\section{Statistical analysis}

Visual acuity was measured using Snellen's chart and was converted to Log MAR for calculation of the means. Values were expressed as mean \pm standard deviation. Lines of vision lost or gained were calculated as Snellen's lines of vision (via Log MAR). The Wilcoxon test was used for continuous variables as age and a chi square test for categorical variables. $\mathrm{P}<0.05$ was considered statistically significant. Software used was Stata version 6.0 (StataCorp, College Station, TX) (Winner 1971).

\section{Results \\ Group I - PPV \\ Preoperative data}

The age of the patients ranged between 24 and 65 years with a mean of $46 \pm 12$ years. Six cases had diabetes mellitus (DM) type I and 9 had type 2 . All cases except 1 had previous PRP treatment. The distribution of cases according to indication for surgery was shown in Table 1 . Mean preoperative visual acuity was $0.016 \pm 6.2$ lines (mean Log mar 1.81 \pm 0.62 ) (range between HM and 0.1$)$. Six cases (40\%) had visual acuity of 0.05 or better. Cataract phaco-surgery was done in 2 cases.

\section{Intraoperative data}

Mean surgical time was $93.3 \pm 11.6$ minutes (range 70 to 110 minutes). Mean bleeding frequency was $6.8 \pm 1.5$ times/case (range between 4 and 9 bleeding attacks/case). Intraoperative bleeding was encountered in all cases. Diathermy was used in all cases with frequency of $4.6 \pm 1.2$ times/case (range 2 to 6 times). Dissection under PFCL was used in 8 cases (53.3\%). Iatrogenic breaks were reported in 6 cases (40\%) and relaxing retinotomies in 4 cases $(26.6 \%)$. Regarding intraoperative tamponade, gas was used in 6 cases $(40 \%)$ and silicone oil in 9 cases $(60 \%)$. The use of silicone was related to relaxing retinotomies and multiple breaks in 4 cases and active bleeding neovascularization in 5 cases $(33.3 \%)$.

\section{Postoperative data}

Mean follow up period was $11.7 \pm 3.04$ months (range 7-17 months). Anatomical attachment was achieved in 13 out of 15 cases (86.6\%), 2 cases (13.3\%) detached due to reproliferation. Final visual acuity showed improvement in 12 cases (80\%), stabilized in 2 cases $(13.3 \%)$ and deteriorated in 1 eye $(6.7 \%)$. The mean final visual acuity reached $0.12 \pm 6.7$ lines (mean Log mar $0.91 \pm 0.67$ ) (range HM -0.5). This improvement of mean visual acuity was statistically significant $(\mathrm{p} 1=0.003)$. Four cases $(26.6 \%)$ reached $20 / 50$ or better.

\section{Postoperative complications}

Persistent cataract was reported in 7 cases (46.7\%) (all were silicone oil cases). Two cases (13.3\%) developed gas 
induced cataract which cleared during the first week. Corneal erosions were observed in 5 cases $(33.3 \%)$ in the early postoperative period. Postoperative bleeding was reported in 4 eyes $(26.6 \%)$; 3 cases of which were gas treated $(50 \%$ of gas treated cases), all of them during the first 3 postoperative weeks and in the fourth case bleeding occurred after silicone removal. All cases cleared spontaneously within 3-6 weeks without treatment. Rubeosis was reported in 1 case $(6.7 \%)$ under silicone oil which was associated with peripheral detachment in lower retina.

Subsequent surgeries: Seven cases $(46.7 \%)$ treated with silicone oil had oil removal and cataract surgery between 4-6 months. Two cases with redetachment underwent re-PPV with silicone oil.

\section{Group 2 - PPV + bevacizumab \\ Preoperative data}

The age of the patients ranged between 23 and 57 years with a mean of $44 \pm 11$. Seven cases had DM type I and 8 had type 2 . All cases except 2 had some degree of laser treatment during the course of the disease. The distribution of cases according to indication for surgery was shown in Table 1. Mean preoperative visual acuity was $0.01 \pm 7.3$ lines (mean $\log$ mar $1.99 \pm 0.73$ ), (range between HM and 0.1). Five cases $(30 \%)$ had visual acuity of 0.05 or better. Cataract surgery was done in 3 cases; 2 phaco and 1 ECCE.

There was evident regression of retinal neovascularization (Figure 1) with no significant deterioration of traction between bevacizumab injection and PPV in cases in which the retina could be clinically visualized.

\section{Intraoperative data}

Mean surgical time was $61.6 \pm 14.5$ minutes (range 40-90 minutes). Mean bleeding frequency was $1.9 \pm 1.1$ times/case (range between 0 and 4 bleeding attacks/case).
In 2 operations, there was no bleeding at all. Diathermy was used only in 4 cases with mean frequency of $0.3 \pm 0.46$ times/case (range 0 to 1 times/case). Dissection under PFCL was used in 3 cases (20\%). Iatrogenic breaks were reported in 3 cases $(20 \%)$ and relaxing retinotomies in 2 cases $(13.3 \%)$. Regarding endotamponade, gas was used in 9 cases $(60 \%)$, air was in 3 cases $(20 \%)$ and silicone oil was used in 3 cases $(20 \%)$. The use of silicone was related to multiple breaks in 1 case, relaxing retinotomies in 1 case and relaxing retinotomies with multiple breaks in 1 case.

\section{Postoperative data}

Mean follow up period was $12.9 \pm 3.2$ months (range 7-18 months). Anatomical attachment was achieved in 14 out of 15 cases $(93.3 \%), 1$ case detached due to reproliferation. Final visual acuity showed improvement in 13 cases (86.6\%), no change in 2 cases (13.3\%). The mean final visual acuity reached $0.18 \pm 6.8$ lines (mean log mar $0.75 \pm 0.68$ ) (range between HM and 0.6). This improvement of mean visual acuity was highly significant ( $p<0.001)$. Four cases $(26.6 \%)$ reached 20/50 or better. The difference in the mean visual acuity between the 2 groups was not statistically significant. $(\mathrm{p}=0.52)$

Postoperative complications were persistent cataract reported in 4 cases (26.6\%) (3 in silicone oil cases), and three cases $(20 \%)$ developed gas-induced cataract which cleared during the first week. Corneal erosions were observed in 2 cases $(13.3 \%)$ in the early postoperative period. None of the cases reported postoperative bleeding or rubeosis. Optic nerve pallor was reported in 1 case.

In subsequent surgeries, 3 cases $(20 \%)$ treated with silicone oil had oil removal and cataract surgery at sometime during the postoperative course. One case $(6.7 \%)$ with redetachment underwent re-PPV with cataract surgery and silicone oil.

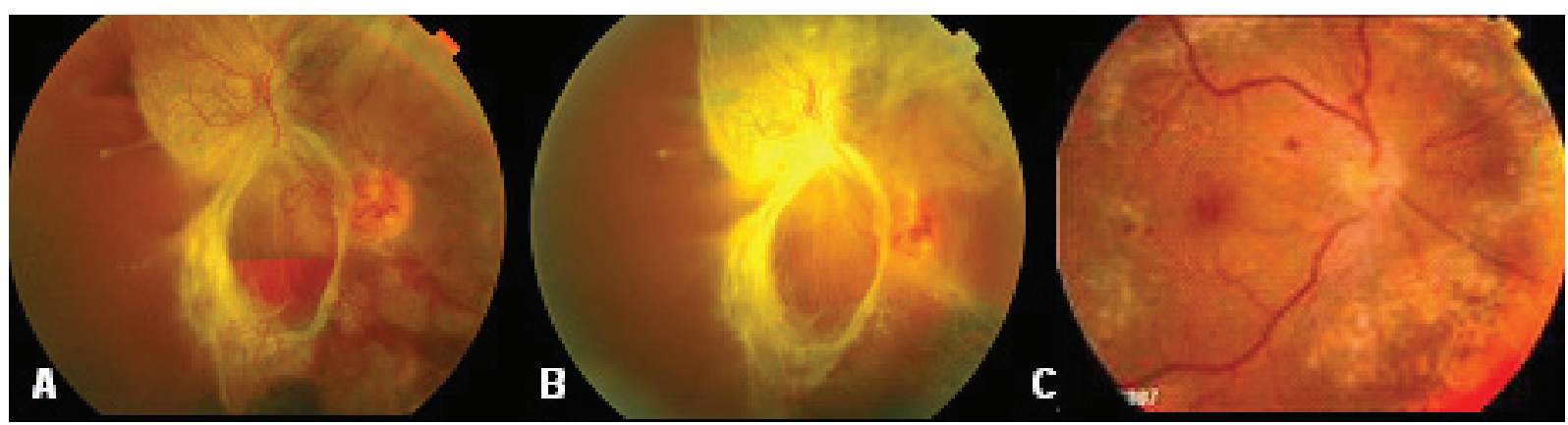

Figure I Fundus photographs of a case in group 2. A before injection of bevacizumab showing vitreous and subhyaloid hemorrhage and tractional retinal detachment. B I week after injection of bevacizumab showing evident regression of retinal neovascularization and C 12 months after vitrectomy with retina attached with near confluent laser marks. 
Table 2 Intraoperative and postoperative data

\begin{tabular}{|c|c|c|c|c|}
\hline & $\begin{array}{l}\text { Group I } \\
\text { PPV }\end{array}$ & $\begin{array}{l}\text { Group } 2 \\
\text { Avastin + PPV }\end{array}$ & $\begin{array}{l}P \text { value } \\
\text { (w) }\end{array}$ & $\begin{array}{l}\text { P value } \\
\text { (chi) }\end{array}$ \\
\hline \multicolumn{5}{|l|}{ Bleeding frequency (times/surgery) } \\
\hline mean (SD)/(range) & $6.8(1.5) /(4-9)$ & $1.9(1.1) /(0-4)$ & $<0.0001$ & \\
\hline \multicolumn{5}{|c|}{ Diathermy frequency (times/surgery) } \\
\hline mean $(\mathrm{SD}) /($ range) & $4.6(1.2) /(2-6)$ & $0.27(0.46) /(0-1)$ & $<0.000$ I & \\
\hline \multicolumn{5}{|l|}{ Surgical time (min.) } \\
\hline mean (SD)/(range) & $93.3(11.6) /(70-110)$ & $61.6(14.5) /(40-90)$ & $<0.0001$ & \\
\hline PFCL use, $n(\%)$ & $8(53.3)$ & $3(20)$ & & \\
\hline Relaxing retinotomy, n (\%) & $4(26.6)$ & $2(13.3)$ & & \\
\hline \multicolumn{5}{|l|}{ Endotamponade, $\mathrm{n}(\%)$} \\
\hline $20 \%$ SF 6 & $6(40)$ & $9(60)$ & & \\
\hline Air & 0 & $3(20)$ & & \\
\hline Silicone oil & $9(60)$ & $3(20)$ & & \\
\hline \multicolumn{5}{|l|}{ VA pre surgery } \\
\hline mean $( \pm$ lines $) /($ range $)$ & $0.016(6.2) /(\mathrm{HM}-0.1)$ & $0.01(7.3) /(\mathrm{HM}-0.1)$ & $\mathrm{P} 2<0.00 \mathrm{I}$ & \\
\hline VA post surgery (final) & & & $P=0.52$ & \\
\hline mean $( \pm$ lines)/(range) & $0.12(6.7) /(\mathrm{HM}-0.5)$ & $0.18(6.8) /(\mathrm{HM}-0.6)$ & $\mathrm{PI}=0.003$ & \\
\hline \multicolumn{5}{|l|}{ VA change, $n(\%)$} \\
\hline Better & $12(80)$ & $13(86.6)$ & & \\
\hline Same & $2(13.3)$ & $2(13.3)$ & & \\
\hline Worse & I (6.7) & 0 & & \\
\hline Primary attachment, n (\%) & $13(86.6)$ & $14(90.3)$ & & 1 \\
\hline \multicolumn{5}{|l|}{ Subsequent surgeries, n (\%) } \\
\hline Cataract + oil removal & $7(46.7)$ & $3(20)$ & & \\
\hline Re-PPV & $2(13.3)$ & I (6.7) & & \\
\hline \multicolumn{5}{|l|}{ Postoperative complications } \\
\hline Cataract, progressive & $7(46.7)$ & $4(26.6)$ & & \\
\hline Cataract, gas-induced & $2(13.3)$ & $3(20)$ & & \\
\hline Postoperative bleeding & $4(26.6)$ & 0 & & \\
\hline Rubeosis & I (6.7) & 0 & & \\
\hline Optic pallor & 0 & I (6.7) & & \\
\hline Corneal erosions & $5(33.3)$ & $2(13.3)$ & & \\
\hline
\end{tabular}

Abbreviations: PPV, pars plana vitrectomy;VA, best corrected visual acuity; ww, Wilcoxon two-sample test; chi, chi square test; PI, preoperative versus finalVA in group I; P2, preoperative versus final VA in group 2; P, p value, visual change between the two groups; SD, standard deviation; min, minutes; PFCL, perfluorocarbon liquid.

The comparative intraoperative and postoperative findings of both groups are summarized in Table 2 .

\section{Discussion}

Pharmacological blockage of VEGF, postulated to be the factor which is primarily responsible for retinal angionesis and vascular permeability, has been investigated extensively (Adamis and Shima 2005). VEGF has been shown to contribute significantly to proliferative diabetic retinopathy Retinal ischemia leads to an increased production of intravitreal VEGF by pigment epithelial cells, pericytes and endothelial cells, while inhibition of VEGF activity via successful panretinal photocoagulation decreases VEGF levels and inhibits retinal neovascularization (Aiello et al 1994).

In this study, intravitreal bevacizumab was injected in an effort to quiet down the fibrovascular proliferation before 
vitrectomy making surgery easier. In the current study, there was marked reduction of intraoperative bleeding in group 2 which was reflected in minimal need to use the endodiathermy. The nearly absence of intraoperative bleeding in Avastin-treated cases can explain the easier dissection of membranes in that group. It is sometimes very difficult to peel clotted blood tightly adherent to the retinal surface which had led to the higher incidence of retinal iatrogenic breaks in group 1. Lee and Abrams (2001) reported that the removal of hemorrhage not only may extend a preexisting retinal break, but also may create new retinal breaks; therefore hemorrhage should be avoided whenever possible. The absence of intraoperative bleeding with evident involution of neovascular complex explains the higher incidence of using gas and air in group 2 in contrast to higher incidence of silicone oil in group 1 . Approximately $60 \%$ of eyes develop recurrent vitreous hemorrhage some time in the postoperative period. Hemorrhage is usually within the first few days after surgery (Liggett et al 1987; Tolentino et al 1989). Two thirds of all hemorrhages occur within the first 6 months (Tolentino et al 1989; Lee and Abrams 2001). Novak and colleagues (1984) reported that vitreous hemorrhage was present on the first postoperative day in $(63 \%)$ of eyes. This early hemorrhage cleared in an average of 9.1 weeks in phakic eyes and 3.4 weeks in aphakic eyes.

In the early postoperative period, the sources of hemorrhage are often difficult to determine. However, most of the surgeons believe that severed fibrovascular membranes are the usual source of bleeding which usually occurs within 1 week of surgery (Meredith 1995; Lee and Abrams 2001). In the Avastin-treated cases, no postoperative bleeding was reported in all cases. A single dose of bevacizumab was found to provide complete VEGF blockade for a minimum of 4 weeks (Beer et al 2006), thus providing a haemostatic effect extending beyond the absorption of the gas. This might have prevented recurrence of bleeding in the early postoperative period in group 2 giving a chance for full PRP and cryotherapy to induce persistent neovascular regression. In group 1 , on the other side, 3 cases out of 6 cases treated with gas tamponade, (50\%), developed early postoperative bleeding while in the remaining 9 cases, the long-term silicone tamponade was the main factor in preventing bleeding in the early postoperative period. The time of surgery was significantly reduced in the avastin group compared to group 1. In a study done by Rizzo and colleagues (2007), the mean surgical time was reduced from 83 minutes to 57 minutes after preoperative injection of Avastin as well as reduction of frequency of intraoperative bleeding and use of endodiathermy. Good visualization due to absence of significant intraoperative bleeding and easier dissection seem to be the reasons for the reduction in the surgical time. Scholda and colleagues (2007), reported an extensive obliteration of neovascularization with only little intraoperative bleeding during surgery after intravitreal Avastin. Chen and Park (2006), after injecting avastin in a case of TRD 1 week before surgery, reported that most epicenters of neovascularization peeled with blunt dissection, suggesting that the recently active neovascular complex had regressed and fibrosed.

In the Avastin-treated cases, no increase in the extent of tractional on the retina was observed. This is because of the short duration between intravitreal injection and surgery which did not exceed 1 week. Scholda and colleagues (2007) reported on 10 cases with advanced PDR treated with avastin 2-3 weeks prior to surgery, the authors reported marked contraction of the remaining fibrous tissue. Arevalo and colleagues (2008), reported that time from injection to progress or development of TRD had a mean of 13 days (range: 3 to 31 days). The authors reported development or progression of TRD in 11 out of 211 eyes (5.2\%) after IVA.

The anatomical and visual results seem comparative in both groups. Vision improved in $87 \%$ in group 2 and $80 \%$ in group 1 with $27 \%$ achieving VA of $20 / 50$ or better in both groups.

Improved vision after vitrectomy is now reported for $60 \%-80 \%$ of eyes with traction macular detachments managed with membrane dissection and PPV. Approximately $20 \%$ of patients achieve vision of at least 20/40 (Meredith 1994). In the Diabetic Retinopathy Vitrectomy Study, patients with active proliferation and good vision preoperatively attained vision of $20 / 40$ or better in $41 \%$ eyes postoperatively (DRVS 1988). In the current study, 5 cases $(33 \%)$ in group 1 and 6 cases $(40 \%)$ in group 2 had preoperative vision between 0.05 and 0.1 . Thompson and colleagues (1986) reported poor initial visual acuity as a predictive factor of poor visual prognosis.

Good anatomical results were achieved in both groups, however in group 2 this was achieved with short term endotamponade (gas or air) in $80 \%$ of cases with less frequency of second surgery while in group 1, silicone oil use was $60 \%$ with subsequent higher rate of resurgery; silicone oil \pm cataract surgery. The choice of silicone oil in this group was strongly related to bleeding tendency which was minimized in group 2 by the IVA. Riedel and colleagues (1990) recommended that oil use be reserved for cases with advanced PDR or intractable PVR. Silicone oil provides the longest term tamponade, and has the additional benefit of noncritical 
posturing. IOP elevation is rarely a problem in the early postoperative period (Flaxel et al 2007). Disadvantages include the certainty of cataract formation and the fact that at some point, the oil must be removed if visual rehabilitation is to be accomplished (Thompson et al 1986). Pearson and colleagues (1993) studied the effect of silicone oil removal following diabetic vitrectomy and concluded that the eventual final visual outcome in these eyes was encouraging in that once cataract and oil were removed; most eyes recovered the acuity achieved soon after initial vitrectomy.

Good anatomical results in both groups can be ascribed to the improvement in vitrectomy equipment. The use of smaller 23 gauge vitrectors offers advantages of being less traumatizing and together with the high speed cutter effect enabled shaving of membranes very close to the retina with minimal trauma. Anterior peripheral retinal cryotherapy combined with cryotherapy of sclerotomy sites might be helpful adjunct procedures in diabetic vitrectomy for inhibition of fibrovascular ingrowth and prevention of recurrent vitreous hemorrhage (Yeh et al 2005). Mason and colleagues (2006) reported that using cryotherapy around sclerotomy sites and endolaser in a near-confluent pattern helps to quell fibrovascular ingrowth and subsequent hemorrhage.

So, in the current study, preoperative IVA was helpful in achieving the surgical and anatomical goals by reducing the time of surgery, reducing the intraoperative and postoperative bleeding and reducing the frequency of using silicone oil with subsequent reduction of second surgery. The combination of therapies and interventions as they improve and evolve offers the potential to revolutionize the approach to the complications of diabetic eye disease.

\section{Disclosure}

The author has no proprietary or conflict of interest in this study.

\section{References}

Adamis AP, Shima DT. 2005. The role of vascular endothelial growth factor in ocular health and disease. Retina, 25:111-18.

Aiello LP, Avery RL, Arrigg PG, et al. 1994. Vascular endothelial growth factor in ocular fluid of patients with diabetic retinopathy and other retinal disorders. $N$ Eng $J$ Med, 331:1480-7.

Aiello LP, Brucker AJ, Chang S, et al. 2004. Evolving guidelines for intravitreal injections. Retina, 24:S3-19.

Arevalo JF, Maia M, Flynn H, et al. 2008. Tractional retinal detachment following intravitreal bevacizumab (Avastin) in patients with severe proliferative diabetic retinopathy. Br J Ophthalmol, 92:213-16.

Avery RL. 2006. Regression of retina and iris neovascularization after Intravitreal bevacizumab (Avastin) treatment. Retina, 26:352-3.

Avery RL, Pieramici DJ, Rabina MD, et al. 2006. Intravitreal bevacizumab (Avastin) for neovascular age related macular degeneration. Ophthalmology, 113:363-2.
Blankenship GW. 1981. Stability of pars plana vitrectomy results for diabetic retinopathy complications: a comparison of 5 year and 6 month postvitrectomy findings. Arch Ophthalmol, 99:1009-12.

Blankenship GW, Machemer R. 1985. Long term diabetic vitrectomy results: report of 10 year follow up. Ophthalmology, 92:503-6.

Beer PM, Wong S, Falk NS, et al. 2006. Vitreous levels of unbound bevacizumab and unbound vascular endothelial growth factor in two human subjects. Program and abstracts of "Cannes Retina Festival"; the joint meeting of the American Society of Retina Specialists and European Vitreoretinal Society, September 9-13, 2006; p. 77.

Chen E, Park CH. 2006. Use of intravitreal bevacizumab as a preoperative adjunct for tractional retinal detachment repair in severe proliferative diabetic retinopathy. Retina, 26:699-70.

de Bustros S, Glaser BM, Michels RG, et al. 1985a. Effect of epsilonaminocaproic acid on postvitrectomy hemorrhage. Arch Ophthalmol, 103:219-21.

de Bustros S, Glaser BM, Johnson MA. 1985b. Thrombin infusion for the control of intraocular bleeding during vitreous surgery. Arch Ophthalmol, 103:837-9.

[DRVS] Diabetic Retinopathy Vitrectomy Study Research Group. 1988. Early vitrectomy for severe proliferative diabetic retinopathy in eyes with useful vision. Results of a randomized trial, Diabetic Vitrectomy Study report 3. Ophthalmology, 95:1307-20.

Davidorf FH, MouserJG, Derick RJ. 2006. Rapid improvement of rubeosis from a single bevacizumab (Avastin) injection. Retina, 26:354-6.

Flaxel CJ, Dustin L, Kim J, et al. 2007. Outcome of diabetic vitrectomy in Latino population. Retina, 27:1274-8.

Grisanti S, Biester S, Peters S, et al. 2006. Intracameral bevacizumab for iris rubeosis. Am J Ophthalmol, 142:158-60.

Liggett PE, Lean JS, Barlow WE, et al. 1987. Intraoperative argon endophotocoagulation for recurrent vitreous hemorrhage after vitrectomy for diabetic retinopathy. Am J Ophthalmol, 103:146-9.

Lee MS, Abrams GW. 2001. Membrane dissection in proliferative diabetic retinopathy. In: Peyman GA, Ameffert S, Conway MD, et al. (eds). Vitreoretinal surgical techniques. London: Martin Dunitz Ltd, pp. 251-66.

Meredith TA. 1994. Current indications for diabetic vitrectomy. In: Lewis H, Ryan SJ (eds). Medical and surgical retina: Advances, controversies and management. St. Louis: Mosby, pp. 290-303.

Manzano RPA, Peyman GA, Khan P, et al. 2006. Testing intravitreal toxicity of bevacizumab (Avastin). Retina, 26:257-61.

Mason JO, Colagross CT, Vail R. 2006. Diabetic vitrectomy: risks, prognosis, future trends. Curr Opin Ophthalmol, 17:281-5.

Novak MA, Rice TA, Michels RG, et al. 1984. Vitreous hemorrhage after vitrectomy for diabetic retinopathy. Ophthalmology, 91:1485-9.

Oshima Y, Sakagauchi H, Gomi F, et al. 2006. Regression of iris neovascularization after Intravitreal injection of bevacizumab in patients of proliferative diabetic retinopathy. Am J Ophthalmol, 142:155-8.

Packer AJ, McCuen BJ, Hutton WL, et al. 1989. Procoagulant effect of intraocular sodium hyaluronate after phakic diabetic vitrectomy: a prospective randomized study. Ophthalmology, 96:1491-4

Pearson RV, McLeod D, Gregor ZJ. 1993. Removal of silicone oil following diabetic vitrectomy. Br J Ophthalmol, 77:204-7.

Riedel KG, Gabel VP, Neubauer L, et al. 1990. Intravitreal silicone injection; complications and treatment of 415 consecutive patients. Graefes Arch Clin Exp Ophthalmol, 228:12-23.

Rizzo S, Genosi-Ebert F, Di Bartolo E, et al. 2007. Injection of intravitreal Avastin before vitrectomy surgery in the treatment of proliferative diabetic retinopathy [abstract]. Program and abstracts of "Cannes Retina Festival"; the joint meeting of the American Society of Retina Specialists and European Vitreoretinal Society, September 9-13, 2007; p. 140.

Spaide RF, Fisher YL. 2006. Intravitreal bevacizumab (Avastin) treatment of proliferative diabetic retinopathy complicated by vitreous hemorrhage. Retina, 26:275-8.

Scholda C, Rainer G, Kriechbaum K, et al. 2007. Preoperative intravitreal injection of bevacizumab in advanced proliferative diabetic retinopathy [abstract]. Joint Congress of SOE/AAO June 9-12, 2007, Vienna, Austria; p. 79. 
Thompson JT, Auer CL, de Bustros S, et al. 1986. Prognostic indicators of success and failure in vitrectomy for diabetic retinopathy. Ophthalmology, 93:290-5.

Tolentino FL, Cajita VN, Gancayco T, et al. 1989. Vitreous hemorrhage after closed vitrectomy for proliferative diabetic retinopathy. Ophthalmology, 96:1495-500.
Winner BJ. 1971. Statistical principles in experimental design. 2nd ed. New York, NY: McGraw-Hill Book Co.

Yeh PT, Yang CM, Yang CH, et al. 2005. Cryotherapy of the anterior retina and sclerotomy sites in diabetic vitrectomy to prevent recurrent vitreous hemorrhage: an ultrasound biomicroscopy study. Ophthalmology, 112:2095-102. 